\title{
Utilização de agentes polinizadores na produção de sementes de cenoura e pimenta doce em cultivo protegido
}

\author{
Warley Marcos Nascimento; Eliana Marília L Gomes; Elizabeth A Batista; Raquel A Freitas \\ Embrapa Hortaliças, C. Postal 218, 70351-970 Brasília-DF; wmn@cnph.embrapa.br
}

\section{RESUMO}

A polinização constitui um processo fundamental para a perpetuação de várias espécies vegetais, e o desenvolvimento dos frutos e das sementes está diretamente relacionado com a polinização das flores. Dessa forma, o objetivo deste trabalho foi verificar a influência de agentes polinizadores na produção e qualidade de sementes de cenoura e de pimenta doce em sistema de cultivo protegido. No ensaio de cenoura utilizaram-se, em telados, os tratamentos de polinização com as abelhas Arapuá (Trigona spinipes), Jataí (Tetragonisca angustula), Tibuna (Nannotrigona (Scaptotrigona) bipunctata), moscas (Musca domestica), polinização manual e livre (fora do telado). No ensaio de pimenta doce, utilizaram-se os tratamentos de polinização com as abelhas Arapuá (Trigona spinipes), Jataí (Tetragonisca angustula), Marmelada (Frieseomellita varia), polinização manual e livre (auto fecundação). A utilização de abelhas Jataí e Tibuna apresentou grande potencial para a produção de sementes de cenoura em condições de cultivo protegido. Para a produção de sementes de pimenta doce não há necessidade de utilização de agentes polinizadores, no entanto a presença desses agentes aumenta o peso dos frutos. A qualidade fisiológica das sementes das duas espécies não foi influenciada pelos diferentes tipos de polinização.

Palavras chave: Capsicum annuum, Daucus carota, polinização, qualidade fisiológica de sementes.

\begin{abstract}
Influence of pollinators on seed production and quality of carrot and sweet pepper in a greenhouse

Pollination is a fundamental process for the perpetuation of various plant species, and the development of fruits and seeds is directly related to the pollination of flowers. Thus, the aim of this study was to assess the influence of pollinators on seed production and quality of carrot and sweet pepper under greenhouse conditions. In the carrot study, plants were grown in cages, and the following treatments were used: pollination with Arapuá (Trigona spinipes) bees, Jataí (Tetragonisca angustula) bees, Tibuna (Nannotrigona (Scaptotrigona) bipunctata) bees, flies (Musca domestica), hand pollination, and free pollination (outside the greenhouse). In the sweet pepper study, the following treatments were used: pollination with Arapuá (Trigona spinipes) bees, Jataí (Tetragonisca angustula) bees, Marmelada (Frieseomellita varia) bees, hand pollination and free (self fertilization). The use of Jataí and Tibuna bees showed a great potential for carrot seed production in greenhouse conditions. For the production of sweet pepper seeds the use of pollinators is not necessary, but the presence of these agents increases the fruit weight. The physiological seed quality of both species was not influenced by different types of pollination.
\end{abstract}

Keywords: Capsicum annuum, Daucus carota, pollination, seed physiological quality.

\section{(Recebido para publicação em 14 de abril de 2011; aceito em 31 de julho de 2012) (Received on April 14, 2011; accepted on July 31, 2012)}

\begin{abstract}
A polinização constitui-se em um fator de produção fundamental na condução de muitas culturas agrícolas ao redor do mundo. Além do aumento no pegamento dos frutos, contribui para a melhoria da qualidade dos mesmos, diminuindo os índices de malformação; aumenta ainda, o teor de óleos e outras substâncias extraídas dos frutos; encurta o ciclo de certas culturas agrícolas e, ainda, uniformiza o amadurecimento dos frutos, reduzindo as perdas na colheita. Uma polinização eficiente pode também contribuir para a melhoria da qualidade fisiológica das sementes produzidas. A polinização pode ocorrer de duas formas: cruzada, quando o pólen de uma flor chega à outra flor da mesma espécie em plantas diferentes; e autopolinização, quando ocorre a transferência do pólen
\end{abstract}

da antera para o estigma de uma mesma flor, ou de outra flor na mesma planta.

Os principais agentes polinizadores são o vento (anemofilia) e os insetos (entomofilia). O homem, outros animais (zoofilia) e a água (hidrofilia) também podem agir na polinização (Marcos Filho, 2005). Estima-se que aproximadamente $73 \%$ das espécies vegetais cultivadas no mundo sejam polinizadas por alguma espécie de abelha (Freitas, 2006). Portanto, dos insetos polinizadores, os mais eficientes são as abelhas, motivo pelo qual é recomendada a manutenção de colméias próximas aos campos de produção de sementes de plantas alógamas (Hawthorn et al., 1956 in Castellane, 1980).

A deficiência de polinização tem sido apontada como uma das causas da baixa produção de sementes em diferentes espécies. Por exemplo, em cebola, para uma eficiente polinização e consequente produção de sementes, torna-se necessário a presença de insetos polinizadores, principalmente as abelhas [Apis mellifera e Trigona spinipes (abelha cachorro, irapuá, arapuá)] e as moscas domésticas (Witter \& Blochtein, 2003; Oliveira, 2005). A abelha Trigona spinipes tem sido utilizada em programas de melhoramento genético de cenoura e cebola (Nascimento et al., 2005).

Acenoura por ser uma planta alógama, necessita de insetos polinizadores para produção de sementes. Estima-se que existam cerca de 334 espécies de insetos potencialmente polinizadores de plantas 
de cenoura, pertencentes a 72 famílias (Bohart \& Nye, 1960 in Castellane, 1980). As umbelas de cenoura são compostas por inflorescências de coloração clara, que somadas à presença de nectários florais, atraem os insetos responsáveis pela realização da polinização (Vieira et al., 2005).

Embora o pimentão seja considerado uma planta autógama, essa espécie beneficia-se da polinização realizada pela abelha Melipona subnitida (Jandaíra), produzindo frutos significativamente mais pesados e mais largos, além de aumentar a produção e a qualidade das sementes. Portanto, Melipona subnitida pode ser considerada uma polinizadora eficiente de pimentão cultivado, por exemplo, em condições de cultivo protegido (Cruz et al., 2005). Efeitos positivos da polinização por abelhas na qualidade dos frutos de pimenta doce foram observados por Jarlan et al. (1997).

A polinização tem importância direta na formação e produção dos frutos, podendo uma polinização deficiente causar deformações, baixo pegamento e baixa produção de frutos. O pegamento de frutos e a produtividade estão relacionados diretamente com a disponibilidade de pólen e a presença de insetos polinizadores na área de cultivo. No entanto, a utilização de agentes polinizadores em áreas cultivadas é uma atividade complexa, exige bons conhecimentos sobre fisiologia de plantas, necessidade de polinização da cultura em questão, eficiência polinizadora do inseto a ser utilizado e o controle do ambiente da área a ser polinizada.

Assim, o objetivo deste trabalho foi verificar a influência de agentes polinizadores na produção de sementes de cenoura e pimenta doce, visando um possível aumento na produção de frutos de pimenta, bem como na produção e qualidade de sementes.

\section{MATERIAL E MÉTODOS}

Estudo 1: Polinização em cenou$\boldsymbol{r a}$ - Realizou-se experimento visando avaliar a influência de diferentes polinizadores na produção de sementes de cenoura (Daucus carota), em campo de produção de sementes básicas de cenoura cultivar Brasília, localizado na área experimental da Embrapa Hortaliças, em Brasília (DF), em 2005.

O campo foi instalado, utilizando-se raízes vernalizadas transplantadas em espaçamento de 0,80 m entre linhas e $0,30 \mathrm{~m}$ entre raízes. As parcelas experimentais constaram de telados de $12 \mathrm{~m}^{2}$, instalados antes do início da floração. Utilizaram-se os seguintes tratamentos: polinização com abelhas Arapuá (Trigona spinipes), Jataí (Tetragonisca angustula) e Tibuna (Nannatrigona (Scaptotrigona) bipunctata), com moscas (Musca domestica), polinização manual e polinização livre (testemunha). Para a polinização manual, umbelas foram diariamente movimentadas com as mãos. Para o tratamento polinização livre, utilizou-se parcelas sem telado. $\mathrm{O}$ experimento foi conduzido no delineamento inteiramente casualizado, com três repetições.

As colméias e as moscas provenientes de criadouros foram colocadas no interior dos telados por ocasião do florescimento das plantas, sendo que as moscas, devido ao curto período de vida, foram repostas a cada dois dias. Os insetos foram alimentados diariamente com solução açucarada.

Após a maturação (cerca de 50 dias após a antese) das sementes, colheram-se aleatoriamente 10 umbelas primárias e 30 umbelas secundárias de cada parcela. As umbelas primárias e secundárias foram colocadas separadamente na sala de secagem a $32^{\circ} \mathrm{C}$, por 24 horas. Em seguida, as sementes foram trilhadas e desaristadas manualmente. Após o beneficiamento, determinou-se a produção de sementes produzidas nas umbelas primárias e secundárias de cada tratamento.

Estudo 2: Polinização em pimenta doce - Para avaliar a influência de diferentes polinizadores na produção de sementes de pimenta doce (Capsicum annuum) cultivar Agronômico 11, realizou-se um experimento no campo experimental da Embrapa Hortaliças, no período de setembro de 2006 a abril de 2007.

As mudas de pimenta foram transplantadas para vasos de cinco litros, 50 dias após a semeadura e mantidos em telado com as laterais abertas. Foram utilizados os seguintes tipos de poliniza- ção: manual; livre; com abelhas Arapuá (Trigona spinipes); Jataí (Tetragonisca angustula) e Marmelada (Frieseomellita varia). $\mathrm{O}$ experimento foi instalado no delineamento inteiramente casualizado com três repetições e 16 plantas (vasos) por parcela. As parcelas que receberam as abelhas foram cobertas por telados individuais.

Todas as flores, bem como os frutos já desenvolvidos antes do início da aplicação dos tratamentos foram eliminados, para iniciar o experimento. As abelhas foram colocadas nos telados em 22/12/2006 e permaneceram até $26 / 01 / 2007$. Os insetos foram alimentados diariamente com solução açucarada colocada em caixas de plástico $(11 \times 11 \times 3,5 \mathrm{~cm})$ dentro dos telados. O tratamento com polinização manual foi realizado diariamente no mesmo período dos outros tratamentos, em que as abelhas permaneceram nos telados.

Após o período de permanência das abelhas nos telados e também da polinização manual, todos os frutos já formados foram etiquetados, visando a colheita apenas dos frutos desenvolvidos nesse período.

Foram feitas contagens do número de frutos presentes no terço médio da planta, em oito plantas de cada parcela. Foram realizadas oito colheitas dos frutos, em intervalos semanais, sendo que a primeira colheita ocorreu aos 70 dias após o transplantio das mudas. Foram feitas contagens do número de frutos colhidos por parcela e determinados o comprimento dos mesmos. Os frutos colhidos permaneceram em repouso por sete dias, em local fresco e arejado e, em seguida, foi determinada a massa dos mesmos. As sementes foram extraídas por via seca e colocadas em peneiras plásticas e secas em uma sala de pré-secagem por 24 horas a $32^{\circ} \mathrm{C}$. O beneficiamento foi feito em um soprador pneumático. Após o beneficiamento, foi determinada a produção de sementes por colheita.

As sementes dos dois ensaios foram submetidas aos seguintes testes e determinações, sendo que as sementes de cenoura foram avaliadas por ordem de umbelas: a) Massa de 100 sementes (gramas) (obtido com quatro repetições de 100 sementes, em balança analítica 
com precisão de três casas decimais); b) Germinação [conduzido conforme metodologia descrita nas Regras para Análise de Sementes (Brasil, 2009)]; c) Primeira contagem (realizada conjuntamente com o teste de germinação, contabilizando-se o número de plântulas normais presentes no sétimo dia após o início do teste); d) Envelhecimento acelerado: utilizou-se a metodologia descrita a seguir: foram distribuídas quatro gramas de sementes sobre tela de alumínio, inserida no interior de caixas de plástico $(11 \times 11 \times 3,5 \mathrm{~cm})$, contendo ao fundo, $40 \mathrm{~mL}$ de solução saturada de $\mathrm{NaCl}$ (40 g de $\mathrm{NaCl} / 100 \mathrm{~mL}$ de água). As caixas contendo as sementes foram fechadas e mantidas a $41^{\circ} \mathrm{C}$, por 72 horas. Após esse período, as sementes foram submetidas ao teste de germinação, conforme descrito anteriormente. A avaliação foi realizada aos sete dias após a instalação do teste; e) emergência das plântulas: feita em casa de vegetação com quatro subamostras de 50 sementes de cada tratamento, as quais foram semeadas em bandejas multicelulares de poliestireno expandido (isopor), contendo 200 células preenchidas com substrato comercial. As bandejas foram mantidas em casa de vegetação e a avaliação das plântulas foi realizada aos quatorze dias após a semeadura.

Os dados expressos em porcentagem foram transformados em arc sen $(\mathrm{x} / 100)^{1 / 2}$ e os demais em raiz $(\mathrm{x}+1)$. Além da análise de variância, os dados foram submetidos ao teste de Tukey, a $5 \%$ de probabilidade, para comparação múltipla de médias.

\section{RESULTADOS E DISCUSSÃO}

Estudo 1- Polinização em cenoura - O tratamento utilizando abelha Arapuá como agente polinizador de cenoura apresentou a menor produção de sementes provenientes de umbelas primárias (Tabela 1), não diferindo, no entanto, dos tratamentos com abelhas Jataí e Tibuna, mosca e polinização manual. Quanto à produção de sementes das umbelas secundárias verificaram-se diferenças significativas entre a utilização da abelha Arapuá e os tratamentos utilizando mosca, polinização livre e manual. À semelhança do que ocorreu para as umbelas de primeira ordem, a abelha Arapuá reduziu a produção de sementes provenientes de umbelas secundárias (Tabela 1).

$\mathrm{Na}$ produção total de sementes (umbelas primárias + secundárias),

Tabela 1. Produção de sementes de cenoura, provenientes de umbelas de ordens primárias (UP) e umbelas secundárias (US) e massa de 100 sementes, utilizando diferentes métodos de polinização (carrot seed production from primary umbel (UP) and secondary umbel (US) and seed mass using different pollination methods). Brasilia, Embrapa Hortaliças, 2005.

\begin{tabular}{lccccc}
\hline \multirow{2}{*}{ Tratamentos } & \multicolumn{2}{c}{ Produção de sementes* $(\mathbf{g})$} & & \multicolumn{2}{c}{ Massa de 100 sementes (g) } \\
\cline { 2 - 3 } \cline { 6 - 7 } \cline { 5 - 6 } Arapuá & UP & US & & UP & US \\
\hline Jataí & $45 \mathrm{~b}$ & $46 \mathrm{~b}$ & & $0,276 \mathrm{a}$ & $0,259 \mathrm{a}$ \\
Tibuna & $72 \mathrm{ab}$ & $73 \mathrm{ab}$ & & $0,233 \mathrm{a}$ & $0,188 \mathrm{a}$ \\
Mosca & $61 \mathrm{ab}$ & $79 \mathrm{ab}$ & & $0,243 \mathrm{a}$ & $0,261 \mathrm{a}$ \\
Manual & $62 \mathrm{ab}$ & $106 \mathrm{a}$ & & $0,236 \mathrm{a}$ & $0,212 \mathrm{a}$ \\
Livre & $64 \mathrm{ab}$ & $83 \mathrm{a}$ & & $0,245 \mathrm{a}$ & $0,239 \mathrm{a}$ \\
\hline CV $(\%)$ & $88 \mathrm{a}$ & $107 \mathrm{a}$ & & $0,252 \mathrm{a}$ & $0,211 \mathrm{a}$ \\
\hline
\end{tabular}

*Produção refererente a 10 umbelas primárias e 30 secundárias (seed production obtained from 10 primary and 30 secondary umbels); Médias seguidas de mesma letra na coluna não diferem entre si pelo teste de Tukey, $\mathrm{p}<0,05$ (means followed by the same letter in the column were not significantly different according to Tukey's test, $\mathrm{p}<0.05$ ).

Tabela 2. Número de frutos presentes no terço médio das plantas, número de frutos total, massa de frutos e massa de sementes de pimenta doce 'Agronômico 11', de acordo com o método de polinização (number of fruits in the middle third of the plant, number of fruits, weight of fruits and seed mass of 'Agronômico 11' sweet-pepper according to the pollination methods). Brasilia, Embrapa Hortaliças, 2007.

\begin{tabular}{lcccc}
\hline Tratamento & $\begin{array}{c}\text { Frutos no terço } \\
\text { médio }\left(\mathbf{n}^{\mathbf{0}}\right)\end{array}$ & $\begin{array}{c}\text { Frutos } \\
\text { colhidos }\left(\mathbf{n}^{\mathbf{0}}\right)\end{array}$ & $\begin{array}{c}\text { Massa de frutos } \\
\text { colhidos }(\mathbf{g})\end{array}$ & $\begin{array}{c}\text { Massa de } \\
\text { sementes }(\mathbf{g})\end{array}$ \\
\hline Arapuá & $9,12 \mathrm{a}$ & $136,0 \mathrm{a}$ & $3790,57 \mathrm{a}$ & $46,64 \mathrm{a}$ \\
Jataí & $7,70 \mathrm{a}$ & $134,3 \mathrm{a}$ & $3182,90 \mathrm{a}$ & $37,06 \mathrm{ab}$ \\
Marmelada & $8,37 \mathrm{a}$ & $127,3 \mathrm{a}$ & $3059,30 \mathrm{a}$ & $32,52 \mathrm{ab}$ \\
Manual & $4,25 \mathrm{~b}$ & $65,7 \mathrm{~b}$ & $1934,30 \mathrm{~b}$ & $29,27 \mathrm{~b}$ \\
Livre & $10,20 \mathrm{a}$ & $148,3 \mathrm{a}$ & $3503,90 \mathrm{a}$ & $42,57 \mathrm{a}$ \\
\hline CV (\%) & 6,40 & 10,36 & 9,30 & 11,79 \\
\hline
\end{tabular}

Médias seguidas de mesma letra na coluna não diferem entre si pelo teste de Tukey, $p<0,05$ (means followed by the same letter in the column were not significantly different according to Tukey's test, $\mathrm{p}<0.05$ ).

Tabela 3. Massa de 100 sementes (MCS), germinação (G), primeira contagem (PC), envelhecimento acelerado (EA) e emergência das plântulas (EP) de pimenta doce 'Agronômico 11', em casa de vegetação, de acordo com o método de polinização (seed mass, seed germination (G), first count (PC), accelerated aging (EA) and seedling emergence (EP) of sweet-pepper 'Agronômico 11', under greenhouse conditions, according to the pollination methods). Brasília, Embrapa Hortaliças, 2007.

\begin{tabular}{lccccc}
\hline Tratamentos & MCS $(\mathbf{g})$ & $\mathbf{G ~} \mathbf{( \% )}$ & $\mathbf{P C}(\boldsymbol{\%})$ & $\mathbf{E A ~}(\%)$ & $\mathbf{E P}(\boldsymbol{\%})$ \\
\hline Arapuá & $0,586 \mathrm{a}$ & $93 \mathrm{a}$ & $83 \mathrm{a}$ & $47 \mathrm{a}$ & $81 \mathrm{a}$ \\
Jataí & $0,608 \mathrm{a}$ & $94 \mathrm{a}$ & $76 \mathrm{a}$ & $41 \mathrm{ab}$ & $80 \mathrm{a}$ \\
Marmelada & $0,586 \mathrm{a}$ & $89 \mathrm{a}$ & $75 \mathrm{a}$ & $30 \mathrm{ab}$ & $80 \mathrm{a}$ \\
Manual & $0,594 \mathrm{a}$ & $85 \mathrm{a}$ & $76 \mathrm{a}$ & $25 \mathrm{~b}$ & $78 \mathrm{a}$ \\
Livre & $0,575 \mathrm{a}$ & $87 \mathrm{a}$ & $68 \mathrm{a}$ & $23 \mathrm{~b}$ & $77 \mathrm{a}$ \\
\hline CV $(\%)$ & 4,31 & 5,66 & 10,40 & 12,85 & 6,08 \\
\hline
\end{tabular}

Médias seguidas de mesma letra na coluna não diferem entre si pelo teste de Tukey, $\mathrm{p}<0,05$ (means followed by the same letter in the column were not significantly different according to Tukey's test, $\mathrm{p}<0.05)$. 
verificou-se que o tratamento com abelha Arapuá mostrou-se inferior aos tratamentos com mosca e polinização livre (Tabela 1). A abelha arapuá proporcionou uma menor produção de sementes, obtendo apenas $91 \mathrm{~g}$ e os tratamentos com moscas e livre, proporcionaram produções de 168 g e 195 g, respectivamente. A maior produção de sementes ocorreu com o emprego da polinização livre (controle), possivelmente devido ao maior número de agentes polinizadores que ocorreu no campo aberto. Vale salientar que existem relatos de cerca de 334 espécies de insetos polinizadores nesta espécie (Bohart \& Nye, 1960 in Castellane, 1980).

Em condições controladas, dentro dos telados, a maior produção de sementes obtida com a utilização de moscas foi devida possivelmente à reposição das mesmas a cada dois dias, aumentando assim a eficiência na polinização. Em adição a isto, o fato da mosca ser bastante pilosa, pode torná-la mais eficiente na polinização quando comparada aos insetos testados no presente ensaio.

A massa de 100 sementes de cenoura dos diferentes tratamentos foi semelhante, independente da ordem das umbelas (Tabela 1). De modo geral, as sementes provenientes de umbelas primárias mostraram-se numericamente superiores às sementes de umbelas secundárias, corroborando com os resultados obtidos por Nascimento (1991).

Com relação à germinação e vigor das sementes, não foi observada diferença significativa entre os tratamentos, demonstrando assim a não influência destes polinizadores sobre a qualidade fisiológica das sementes de cenoura (dados não apresentados). Como era esperado, apesar de não ter sido feito análise estatística, observou-se um maior valor numérico na primeira contagem, germinação, envelhecimento acelerado e emergência das plântulas das sementes provenientes das umbelas primárias quando comparadas com as umbelas secundárias. Sementes oriundas de umbelas de menor ordem apresentam maior qualidade fisiológica em relação às demais ordens (Nascimento, 1991; Cardoso, 2000).

Estudo 2 - Polinização em pimenta - O maior tamanho de fruto foi obtido na sétima colheita para o tratamento utilizando a abelha Marmelada como agente polinizador. De forma similar, Shipp et al. (1994) verificaram que os polinizadores Osmia cornifrons, Megachile rotundata e Apis mellifera também proporcionaram incrementos no tamanho dos frutos de pimenta doce. Jarlan et al. (1997) por sua vez, obtiveram frutos pesados e longos de pimenta doce, utilizando a mosca Eristalis tenax como polinizadora. No entanto, Cruz et al. (2005) observaram que o comprimento do fruto não diferiu entre os tratamentos com polinização manual, polinização elétrica e polinização com abelha solitária Megachile rotundata. Segundo esses autores, pelo fato da forma do fruto da pimenta doce variar de acordo com a cultivar, é possível que frutos de algumas cultivares possam ser afetados diferentemente com o tipo de polinização que eles recebem.

As plantas de pimenta doce polinizadas manualmente apresentaram os menores números de frutos no terço médio da planta e de frutos colhidos, além de menor massa de frutos e de sementes (Tabela 2). Esses resultados discordam dos obtidos por Godoy et al. (2006), os quais obtiveram frutos de pimentão com a mesma massa nas polinizações manual e livre. Alguns autores têm mostrado que polinizações inadequadas aumentam porcentagens de frutos mal formados. Vários fatores podem influenciar a eficiência da polinização como quantidade de pólen insuficiente, baixa viabilidade ou incompatibilidade do pólen, condições ambientais e nutricionais, déficit hídrico, dentre outros fatores (Free, 1993). Dag \& Kammer (2001) obtiveram incrementos em peso de frutos, com a utilização das abelhas Apis mellifera ou Bombus terrestris para polinizar pimenta doce em estufas, comparada com um tratamento sem abelhas.

O tratamento utilizando a abelha Arapuá, Jataí e Marmelada, além da polinização livre não diferiram entre si (Tabela 2). Porém, Cruz et al. (2005) verificaram que o número de sementes por frutos diferiu significantemente entre os tratamentos com polinização manual, polinização elétrica e polinização com abelha solitária Megachile rotundata, sendo que polinização manual e poli- nização com abelhas apresentaram um maior número de sementes, comparados à polinização livre. Resultados similares foram observados por Meisels \& Chiasson (1997) na polinização de flores de pimenta doce por B. impatiens, em estufas. Godoy et al. (2006) verificaram que a produção de sementes por fruto foi maior nos frutos que tiveram a sua polinização natural, embora não tenha havido diferença significativa em relação às polinizações manuais realizadas no dia da emasculação e um dia após (no dia da antese). Obtiveram-se menor produção no tratamento com polinização manual realizado três dias após a emasculação.

A germinação e o vigor das sementes de pimenta doce avaliados pelos testes de primeira contagem e emergência de plântulas não mostraram diferenças significativas entre os diferentes tratamentos utilizados (Tabela 3). Entretanto, o teste de envelhecimento acelerado indicou as sementes oriundas do tratamento com Arapuá, Jataí e Marmelada como as de maior vigor (Tabela 3).

Os dados obtidos neste estudo permitem concluir que para a produção de sementes de pimenta doce não há necessidade de utilização de agentes polinizadores; no entanto, a presença desses agentes aumenta o peso dos frutos. A utilização das abelhas Jataí e Tibuna apresentam um grande potencial para a produção de sementes de cenoura em condições de cultivo protegido. Vale salientar que estas duas espécies são facilmente manejadas e de menor custo quando comparadas com moscas e/ou polinização manual, por exemplo. A qualidade fisiológica (germinação e vigor) das sementes de cenoura e pimenta doce não foi influenciada pelos polinizadores e/ou métodos de polinização.

\section{REFERÊNCIAS}

BRASIL. 2009. Ministério da Agricultura. Regras para análise de semente. Secretaria Nacional de Defesa Agropecuária, 399p.

CARDOSO AII. 2000. Produção e qualidade de sementes de cenoura das cultivares Brasília e Carandaí. Bragantia 59: 77-81.

CASTELLANE PD. 1980. Produção de sementes de cenoura (Daucus carota L.). In: MULLER JJV; CASALI VWD (eds). Seminários de Olericultura. $2^{\mathrm{a}}$ ed. v.1 Viçosa, UFV, p.36-76.

CRUZ DO; FREITAS BM; SILVA LA; SILVA EMS; BOMFIM IGA. 2005. Pollination 
efficiency of the stingless bee Melipona subnitida on greenhouse sweet pepper. Pesquisa Agropecuária Brasileira 40: 1197. 1201.

DAG A; KAMMER Y. 2001. Comparison between the effectiveness of honey bee (Apis mellifera) and bumble bee (Bombus terrestris) as pollinators of greenhouse sweet pepper (Capsicum annuum). American Bee Journal 141: 447-448.

FREE JB. 1993. Insect pollination of crops. 2 ed. London: Academic, 684p.

FREITAS BM. 2006. Polinizadores e polinização: o valor econômico da conservação. Disponível em: http://www.reacao.com.br/programa sbpc57ra/sbpccontrole/textos/brenofreitas.htm Acesso em: 20/01/2006.

GODOY MC; GODOY AR; CARDOSO AII. 2006. Influência do estádio de maturação da flor na produção de sementes de pimentão com polinização manual. Bragantia 65: 83-87.

JARLAN A; OLIVEIRA D; GINGRAS J. 1997.
Pollination of sweet pepper (Capsicum annuит L.) in greenhouse by the syrphid fly Eristalis tenax L. Acta Horticulturae 437: 335-340.

MEISELS S; CHIASSON H. 1997. Effectiveness of Bombus impatiens $\mathrm{Cr}$. as pollinators of greenhouse sweet peppers (Capsicum annuum L.). Acta Horticulturae 437: 425-430.

NASCIMENTO WM. 1991. Efeito da ordem das umbelas na produção e qualidade de sementes de cenoura. Revista Brasileira de Sementes 13: 131-133.

NASCIMENTO WM; VIEIRA JV; OLIVEIRA VR. 2005. Utilização de Trigona spinipes em programas de melhoramento genético de cebola e cenoura. Encontro da Sociedade Brasileira de Melhoramento de Plantas Regional DF, 1. 2005., Anais... Brasília: Embrapa Recursos Genéticos e Biotecnologia, 2005 (CD-ROM) (Documentos 144).

OLIVEIRA VR. 2005. Produção de sementes de cebola. In: CURSO SOBRE TECNOLOGIA DE PRODUÇÃO DE SEMENTES DE
HORTALIÇAS, 5., 2005, Brasília. Palestras... Brasília: Embrapa Hortaliças, 2005 (CDROM).

SHIPP JL; WHITFIELD GH; PAPADOPOULOS AP. 1994. Effectiveness of the bumble bee, Bombus impatiens Cr. (Hymenoptera: Apidae), as a pollinator of greenhouse sweet pepper. Scientia Horticulturae 57: 29-39.

MARCOS FILHO, J. 2005. Fisiologia de sementes de plantas cultivadas. Piracicaba: FEALQ, $495 \mathrm{p}$

VIEIRA JV; NASCIMENTO WM; MAROUELLI WA. 2005. Produção de sementes de cenoura. In: CURSO SOBRE TECNOLOGIA DE PRODUÇÃO DE SEMENTES DE HORTALIÇAS, 5., 2005, Brasília. Palestras... Brasília: Embrapa Hortaliças, 2005 (CDROM).

WITTER S; BLOCHTEIN B. 2003. Efeito da polinização por abelhas e outros insetos na produção de sementes de cebola. Pesquisa Agropecuária Brasileira 38: 1399-1407. 